\title{
Telomere length comparison between oral cells and blood cells among neonates
}

\author{
Mandar Bhattacharya, Pranami Bhaumik, Subrata Kumar-Dey \\ Department of Biotechnology, Maulana Abul Kalam Azad University of Technology, BF 142, Sector 1, Salt Lake City, \\ Kolkata, West Bengal 700064. E-mail: skdey.makaut@gmail.com \\ Received: 28th May 2018, Revised: 8th October 2018, 3rd January 2019, Accepted: 29th January 2019
}

SUMMARY: Bhattacharya M, Bhaumik P, Kumar-Dey S. Telomere length comparison between oral cells and blood cells among neonates. Turk J Pediatr 2019; 61: 520-524.

Telomere length, measured from blood cells, is commonly used as the standard telomere length of the whole body. The relationship between blood telomere length and oral telomere length is still unclear, especially among neonates. In this study, we measured blood telomere length as well as cheek cells telomere length to find out the overall telomere synchronization in neonates. Children aged 1 month or younger were included in this study. Blood and cheek cells were collected by heel stick method and nylon brush accordingly. Telomere length was measured by Southern blotting. A strong telomere length correlation $(0.77, \mathrm{p}<0.001)$ existed between oral and blood cells which showing telomere length synchronization among different tissue types. Mean telomere length and length variability were significantly higher $(t=3.73, p=0.0004)$ in oral cells. Longer telomere among oral cells can be justified by progenitor cell pool theory. The cause of high telomere length variability in the oral source is not clear although the presence of leukocytes among cheek cells cannot be excluded. Therefore, for telomere length measurement purpose blood should be given preference over the oral source.

Key words: telomere length, neonates, white blood cells, oral cells, cheek cells.

Telomeres are repetitive elements that act as a molecular cap for each chromosomal end. Telomere length is controlled by telomerase enzyme. Telomerase activity is negligible in normal cells, so telomere length gets shortened after each round of replication. Once telomere becomes critically short, cells reach senescence. On the other hand, telomerase is overtly active in immortal cancer cells, maintaining telomere length throughout the cellular lifespan. ${ }^{1}$ This protective property of telomere makes it a suitable molecular aging marker. ${ }^{2}$ Since every living cell in our body has telomere, tissue-specific telomere length difference provides us with new insights about tissue-specific cell senescence pattern. Complex familial telomere length association patterns and telomere length related parental epidemiological studies require lower degrees of telomere length variability in a population. Therefore, selecting a tissue type with lower telomere length variability is important. Peripheral blood is considered as the standard tissue for telomere length measurement. It can be easily collected and provides abundant high-quality DNA. Unfortunately, the blood collection procedure is invasive and uncomfortable.

DNA collection from oral source is gaining popularity especially among children. It is easy to collect, completely painless and provides a high quantity of genomic DNA. However, the majority of studies about telomere length difference between blood and oral source have been carried out among adults. ${ }^{3}$ Little information is available among children, especially newborns. Hence, our goal was to evaluate whether any significant telomere length difference exists between invasive (blood) and non-invasive (cheek cells) sources of tissue collection. 


\section{Material and Methods}

Study Population: Between May 2015 and September 2016 a total number of 41 neonates were selected. Only 30 days of age or younger children were included in this study so that we could negate age-dependent telomere shortening. No premature or postmature babies (small and high gestational age babies) were included in the study. The gestational age range was strictly kept between 37 to 42 weeks. Mothers with chronic or acute autoimmune disorders, diabetes, morbid obesity, cancer and any form of addiction were excluded. Maternal karyotyping was done to check for chromosomal integrity. Paternal age was kept strictly between 24 to 29 years.

\section{Sample collection, DNA isolation, and Southern Blot}

Cheek cells were collected with nylon bristle cytology brush. ${ }^{4}$ Peripheral blood has been collected as per standard recommendation. ${ }^{5}$ Both blood and cheek samples were taken from each individual. Colcimid mediated standard karyotyping procedure was obtained to rule out any chromosomally abnormal neonates. DNA isolation was done by Qiagen QIAmp DNA Mini kit ${ }^{\circledR}$. DNA integrity was checked in $0.8 \%$ agarose gel. Telomere length was measured by Roche Telo TAGGG® telomere length assay kit. In short, isolated DNA was digested with HinfI and RsaI restriction enzymes. DNA fragments were electrophoresed in agarose gel. The gel was then treated with $\mathrm{HCl}$ solution, denaturation solution $(0.5 \mathrm{M} \mathrm{NaOH}, 1.5 \mathrm{M}$ $\mathrm{NaCl})$ and neutralization solution $(0.5 \mathrm{M}$ Tris$\mathrm{HCl}, 3 \mathrm{M} \mathrm{NaCl}, \mathrm{pH}$ 7.5), respectively. Next, DNA fragments were transferred on a nylon membrane by capillary action. We used $20 \mathrm{X}$ SSC transfer buffer in this process. After UV crosslinking, the membrane was hybridized with DIG-labelled telomere-specific probes. Fluorescence activity was recorded by exposing $\mathrm{X}$-ray film to the membrane. Once the film development process was done, telomere length was estimated by densitometric analysis. Densitometric analysis was done using Image-J (National Institute of Health) software and GNU Image Manipulation Programme (GIMP) software. Mean TRF was calculated using the following formula: $\mathrm{L}=\Sigma(\mathrm{ODi}) / \Sigma(\mathrm{ODi}) /(\mathrm{Li})$. Here ODi represents the chemiluminescent signal intensity and $\mathrm{Li}$ represents the length of the TRF fragment at position i. In this study mean TRF and telomere length (TL) bears the same meaning.

\section{Data tabulation and statistical analysis:}

Birth weight, oral TL, white blood cell (WBC) $\mathrm{TL}$, and gestational age were used as continuous variables while sex as a nominal variable. Data were arranged in an Excel sheet where each row represents a separate individual and each column represents one variable. Mean and standard deviation was calculated for each variable. Normality of the data was checked by the Shapiro-Wilk test. Linear regression was done for each TL source separately and suitable correlation method was implemented.

Informed consent was collected in each case. All of our work has been cleared by institutional ethical committee [Ref. MAKAUT: IEC(16-17)/12]. Samples cannot be identified from the data presented here. We do respect and understand the Declaration of Helsinki proposed by the World Medical Association.

\section{Result}

A total of 41 neonates were included in this study, out of which 25 were male and 16 were female. Maternal age varied from 22 to 31 years. Overall mean weight (SD) was 2.98 (0.35) kilograms. The average weight of a male child and female child was 3.03 (0.37) kilograms and 2.9 (0.32) kilograms, respectively. No significant birth weight difference between male and females was observed $(z=-1.203$, $\mathrm{p}=0.229$ ). Telomere length range for WBC was $11.49 \mathrm{~Kb}$ to $12.55 \mathrm{~Kb}$. Telomere length from oral source had a wider range than TL from WBC source, ranging $11.45 \mathrm{~Kb}$ to 12.98 $\mathrm{Kb}$ (Fig. 1). Overall the telomere length in WBC had a mean value of $11.97(0.33) \mathrm{kb}$ and for cheek cells, it was $12.3(0.47) \mathrm{kb}$ as shown in Table I. The mean telomere length difference between WBC and cheek cells was highly significant $(t=3.73, p=0.0004)$ with a high positive correlation (Pearson's coefficient of correlation $0.747, \mathrm{p}<0.001$ ) (Fig. 2). Only 5 cases out of 41 samples we studied exhibited higher WBC TL than oral TL. Both WBC and oral telomere length did not depend on child's sex, birth weight or gestational age $(p=0.336$, 
Table I. Weight, Gestation status and Telomere Length in Studied samples.

\begin{tabular}{lccc}
\hline Characteristics & Male $($ mean $\pm \mathrm{SD})$ & Female $($ mean $\pm \mathrm{SD})$ & Total $($ mean $\pm \mathrm{SD})$ \\
\hline Total number & $\mathrm{N}=25$ & $\mathrm{~N}=16$ & $\mathrm{~N}=41$ \\
Weight (Kilogram) & $3.03 \pm 0.37$ & $2.9 \pm 0.32$ & $2.98 \pm 0.35$ \\
WBC TL (Kb) & $11.94 \pm 0.32$ & $12.02 \pm 0.36$ & $11.97 \pm 0.33$ \\
Oral TL $(\mathrm{Kb})$ & $12.34 \pm 0.4$ & $12.25 \pm 0.55$ & $12.3 \pm 0.47$ \\
Gestational age (weeks) & $38.18 \pm 1.01$ & $39.97 \pm 1.35$ & $39.3 \pm 1.5$ \\
\hline
\end{tabular}

TL: Telomere length

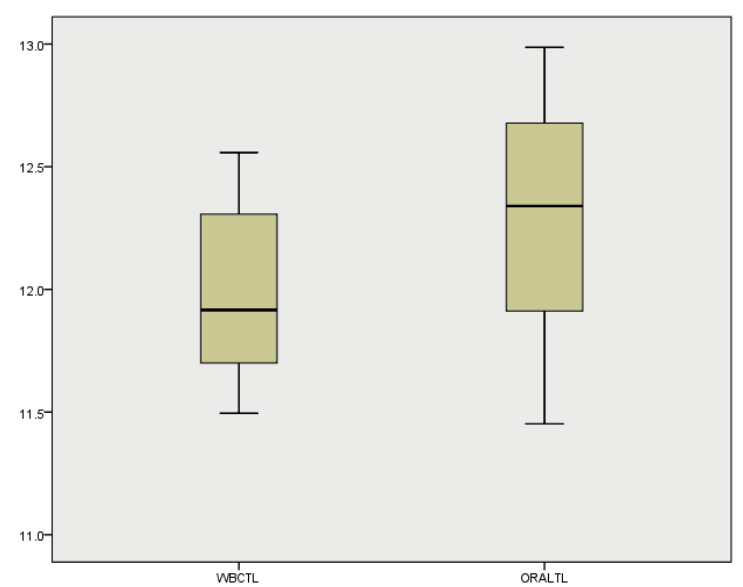

Fig. 1. Box and whisker plot which shows oral telomere length has wider range than whole blood cell telomere length. Y axis represents TRF in $\mathrm{Kb}$.

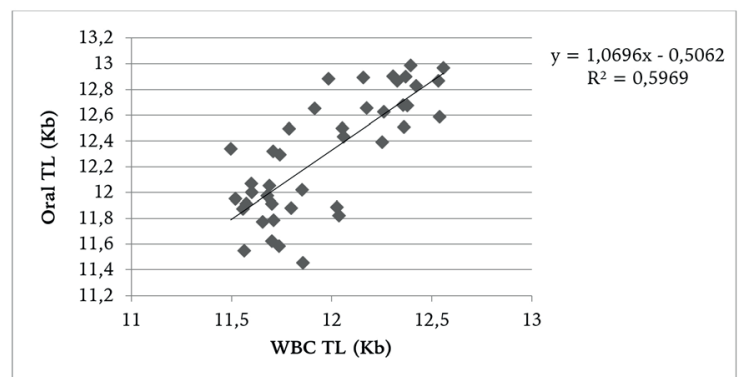

Fig. 2. High correlation exists between oral telomere and whole blood telomere suggesting telomere synchronization.

0.273 and 0.5 for WBC source; $\mathrm{p}=0.574,0.134$ and 0.4 for cheek cell source).

\section{Discussion}

In this study, our goal was to correlate the telomere lengths obtained from cheek cells and blood leukocyte. Three key conclusions can be drawn from the study. Firstly, telomere length from the blood source and oral source are significantly correlated within the same individual. Secondly, we found mean telomere length was higher in cheek cells than WBC and thirdly, telomere length variation (in terms of length range) was higher in oral cells than WBCs among the studied samples.

We did not observe a significant difference between male and female TRF lengths. There were several published reports which showed longer telomere in females than males. ${ }^{6}$ However, our findings fall in line with another study where estrogen-mediated TRF control had been proposed, although we did not measure the hormone level. ${ }^{7}$

Telomere length variation has been reported in different tissues in adults. According to a study, leukocyte had the shortest telomere compared to skin and muscles although TL among tissues is highly correlated. ${ }^{3}$ In another study among adults, no significant correlation has been observed between tongue mucosa and leukocyte TL, although TL in mucosa is significantly shorter than leukocytes and leukocyte telomere is significantly longer than skin telomere. ${ }^{8}$ The same study also proposed a significant level of correlation between leukocytes TL and muscle TL. In new-borns, in a cross-sectional study WBC TL was significantly longer than foreskin $\mathrm{TL}$ and a strong positive correlation exists among WBC TL and foreskin TL. ${ }^{7}$ In utero telomere synchronization models in fetuses demonstrate high linearity in telomere length among different tissue types. ${ }^{9}$ In other words, all tissue types such as both blood and cheek cells exhibit either long or short telomere length in a particular individual. This strong synchrony of telomere length has 
been observed across the species including mammals, canine and birds. ${ }^{10-12}$ Not only are the tissue-specific TLs strongly correlated, but during the aging process, the rate of TL attrition are also similar. Our data support this model as a high level of correlation between WBC TL and cheek cell TL was observed. However, telomere lengths differ in different tissues within a single neonate as well as from different neonatal samples. ${ }^{7}$

Exact TL in humans is somewhat ambiguous as several contradicting reports have been observed. We observed longer telomere in cheek cells compared to WBC cells. This observation of ours contradicts a study which was previously done by Dlouha et al., ${ }^{8}$ but is supported by other studies. ${ }^{13}$ However, as previously mentioned, these studies were done on adult subjects. The reason behind our second observation is not clear at this point. One way to explain this result is via progenitor cell pool expansion and consecutive telomere attrition. Different tissues have different proliferation rates. Highly proliferative tissues need a higher amount of progenitor cells to maintain cellular homeostasis. Progenitor cell pool dynamics proposed by Danieli et al., 3 confirmed that the higher the proliferative nature of tissue, the higher the TL attrition rate in early age, possibly at the foetus stage. ${ }^{14}$ If we take high proliferative nature of blood cells into consideration, progenitor cell pool size must be higher than its oral epithelial counterpart, therefore exhibiting higher TL attrition during early development. So, in uterine determination of higher oral TL length followed by an identical level of TL attrition among tissues after birth, may be the reason why some studies reported higher oral TL among adults.

It is therefore clear that both cheek cell telomere length and blood cell telomere length has the potential to be used as a "genetic aging" marker. Now to answer the question of which one we should use, we need to take a look at our third observation where a wider range of TL values has been observed among oral source. Again, the exact cause of this observation is not clear. One possibility is the presence of different cell types among oral cavity as large amounts of leukocyte cells may be present in saliva. ${ }^{15}$ While collecting cheek epithelial cells from neonates, mixing of saliva eventually occurs, this, in turn, may contribute to the high variability in oral TL.

In children, short telomere length has been associated with congenital desorders, hyperactivity, behavior issues, depression. .,16-19 $^{4}$ Social and economic variables are also directly associated with children's' telomere length thereby introducing premature aging. ${ }^{20,22}$ Hence understanding and measuring the telomere length is crucial not only to determine the biological factors affecting telomere length but also to assess the risk of future premature aging.

We accept some obvious drawbacks in this study. We included children who were overall healthy at the time of the experiment. However, that doesn't mean complete diseasefree condition. Maternal stress status during pregnancy was also unknown. Thirdly, our sample size is relatively low and expansion is required.

This is the first study, to our knowledge, to compare telomere length from blood and oral source among neonates. In conclusion, we found higher TL in oral source than WBCs and TL from both the sources were highly correlated. As both the source can be used for telomere length analysis purpose, low TL variability in WBC makes it a more prudent choice over the oral source. TL analysis among neonates should include both the sources to get a clearer picture.

\section{Funding}

Financial support for this work is provided by the Indian Council of Medical Research (ICMR) [Grant No. 54/10/2012-HUM-BMS].

\section{REFERENCES}

1. Shay JW, Zou Y, Hiyama E, Wright WE. Telomerase and cancer. Hum Mol Genet 2001; 10: 677-685.

2. Mather KA, Jorm AF, Parslow RA, Christensen H. Is telomere length a biomarker of aging? A review. J Gerontol A Biol Sci Med Sci 2011; 66: 202-213.

3. Daniali L, Benetos A, Susser E, et al. Telomeres shorten at equivalent rates in somatic tissues of adults. Nat Commun 2013; 4: 1597. 
4. Kroenke $\mathrm{CH}$, Epel E, Adler N, et al. Autonomic and adrenocortical reactivity and buccal cell telomere length in kindergarten children. Psychosom Med 2011; 73: 533-540

5. Howie SR. Blood sample volumes in child health research: review of safe limits. Bull World Health Organ 2011; 89: 46-53.

6. Benetos A, Okuda K, Lajemi M, et al. Telomere length as an indicator of biological aging: the gender effect and relation with pulse pressure and pulse wave velocity. Hypertension 2001; 37 (2 Pt 2): 381-385.

7. Okuda K, Bardeguez A, Gardner JP, et al. Telomere length in the newborn. Pediatr Res 2002; 52: 377381.

8. Dlouha D, Maluskova J, Kralova Lesna I, Lanska V, Hubacek JA. Comparison of the relative telomere length measured in leukocytes and eleven different human tissues. Physiol Res 2014; 63(Suppl 3): S343-S350.

9. Youngren K, Jeanclos E, Aviv H, et al. Synchrony in telomere length of the human fetus. Hum Genet 1998; 102: 640-643.

10. Gardner JP, Kimura M, Chai W, et al. Telomere dynamics in macaques and humans. J Gerontol A Biol Sci Med Sci 2007; 62:367-374.

11. Benetos A, Kimura M, Labat C, et al. A model of canine leukocyte telomere dynamics. Aging Cell 2011;10: 991-995.

12. Reichert S, Criscuolo F, Verinaud E, Zahn S, Massemin S. Telomere length correlations among somatic tissues in adult zebra finches. PLoS One 2013; 8: e81496.

13. Karlsson AO, Svensson A, Marklund A, Holmlund G. Estimating human age in forensic samples by analysis of telomere repeats. Forensic Sci Int: Genet Suppl Ser 2008; 1: 569-571.
14. Frenck RW Jr, Blackburn EH, Shannon KM. The rate of telomere sequence loss in human leukocytes varies with age. Proc Natl Acad Sci U S A 1998; 95: $5607-$ 5610 .

15. Thiede C, Prange-Krex G, Freiberg-Richter J, Bornhäuser M, Ehninger G. Buccal swabs but not mouthwash samples can be used to obtain pretransplant DNA fingerprints from recipients of allogeneic bone marrow transplants. Bone Marrow Transplant 2000; 25: 575-577.

16. Kostjukovits S, Degerman S, Pekkinen $M$, et al. Decreased telomere length in children with cartilagehair hypoplasia. J Med Genet 2017; 54: 365-370.

17. Gruszecka A, Kopczyński P, Cudziło D, et al. Telomere shortening in Down syndrome patients--when does it start? DNA Cell Biol 2015; 34: 412-417.

18. Anderson ER, Mayes LC. Race/ethnicity and internalizing disorders in youth: a review. Clin Psychol Rev 2010; 30: 338-348.

19. Orth U, Robins RW, Widaman KF, Conger RD. Is low self-esteem a risk factor for depression? Findings from a longitudinal study of Mexican-origin youth. Dev Psychol 2014; 50: 622-633.

20. Feder A, Alonso A, Tang M, et al. Children of lowincome depressed mothers: psychiatric disorders and social adjustment. Depress Anxiety 2009; 26: 513 520 .

21. Mitchell C, Hobcraft J, McLanahan SS, et al. Social disadvantage, genetic sensitivity, and children's telomere length. Proc Natl Acad Sci U S A 2014; 111: 5944-5949.

22. Drury SS, Theall K, Gleason MM, et al. Telomere length and early severe social deprivation: linking early adversity and cellular aging. Mol Psychiatry 2012; 17:719-727. 